\title{
Alterstice
}

Revue internationale de la recherche interculturelle

International Journal of Intercultural Research

Revista International de la Investigacion Intercultural

\section{Familles transnationales défiant les États-nation : quatre lieux d'engagement autour des droits des enfants et des familles}

\section{Gail Mummert}

Volume 3, numéro 1, 2013

Les diversités au coeur de la recherche interculturelle : vers de nouvelles perspectives

URI : https://id.erudit.org/iderudit/1077496ar

DOI : https://doi.org/10.7202/1077496ar

Aller au sommaire du numéro

Éditeur(s)

Alterstice

ISSN

1923-919X (numérique)

Découvrir la revue

Citer cet article

Mummert, G. (2013). Familles transnationales défiant les États-nation : quatre lieux d'engagement autour des droits des enfants et des familles. Alterstice,

3(1), 17-33. https://doi.org/10.7202/1077496ar

\section{Résumé de l'article}

Cet article porte sur certains rapports tissés entre les familles transnationales et les États-nation, analysés comme lieux d'engagement et enjeux politiques. La vie quotidienne de ces groupes de parents, qui organisent leurs activités productives et reproductives à travers une ou plusieurs frontières internationales, est marquée par l'incertitude, le sacrifice et la fluidité. Leurs membres agissent à cheval entre les contraintes dictées par les gouvernements et les opportunités créées par les sujets transnationaux eux-mêmes, qui se voient dotés par les États nation d'un statut ambigu où ils font l'objet de soupçons permanents. À travers notre analyse de quatre événements historiques (lieux d'engagement autour des droits de l'enfant) s'enchaînant depuis la Guerre froide jusqu'à nos jours, nous remettrons en question le stéréotype d'institutions d'État qui surveilleraient constamment les activités et les pratiques de leurs citoyens et résidents, ceux-ci se retrouvant victimes passives des contrôles de celles-là. Utilisant la notion d'interface sociale, nous soulignons, au contraire, les actions menées par les membres des familles transnationales qui, en naviguant à travers les interstices légaux entre au moins deux gouvernements, vont jusqu'à défier leur autorité. 


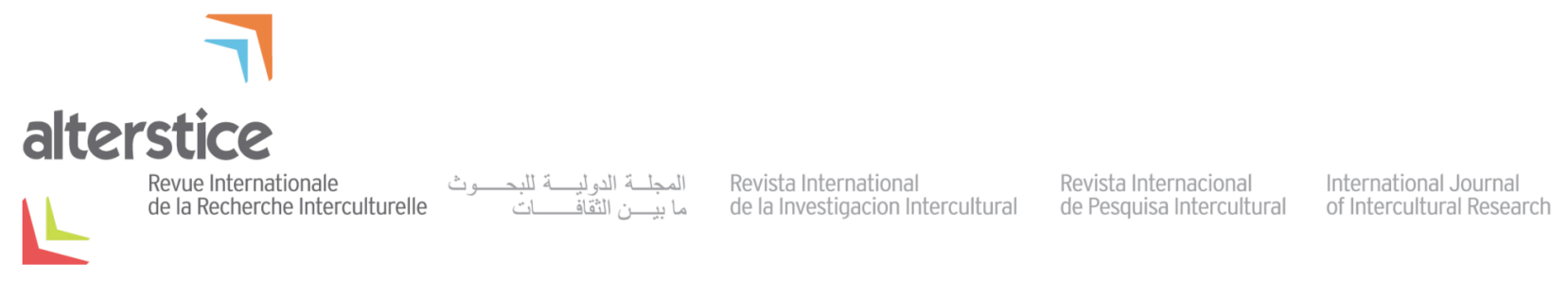

ARTICLE THÉMATIQUE

\section{Familles transnationales défiant les États-nation : quatre lieux d'engagement autour des droits des enfants et des familles}

Gail Mummert ${ }^{1}$

\section{Résumé}

Cet article porte sur certains rapports tissés entre les familles transnationales et les États-nation, analysés comme lieux d'engagement et enjeux politiques. La vie quotidienne de ces groupes de parents, qui organisent leurs activités productives et reproductives à travers une ou plusieurs frontières internationales, est marquée par l'incertitude, le sacrifice et la fluidité. Leurs membres agissent à cheval entre les contraintes dictées par les gouvernements et les opportunités créées par les sujets transnationaux eux-mêmes, qui se voient dotés par les États nation d'un statut ambigu où ils font l'objet de soupçons permanents. À travers notre analyse de quatre événements historiques (lieux d'engagement autour des droits de l'enfant) s'enchaînant depuis la Guerre froide jusqu'à nos jours, nous remettrons en question le stéréotype d'institutions d'État qui surveilleraient constamment les activités et les pratiques de leurs citoyens et résidents, ceux-ci se retrouvant victimes passives des contrôles de celles-là. Utilisant la notion d'interface sociale, nous soulignons, au contraire, les actions menées par les membres des familles transnationales qui, en naviguant à travers les interstices légaux entre au moins deux gouvernements, vont jusqu'à défier leur autorité.

\section{Rattachement de l'auteure \\ ${ }^{1}$ El Colegio de Michoacán, Zamora, Mexique.}

\section{Correspondance}

gailmummert@hotmail.com

\section{Mots clés}

Famille transnationale, maternité et paternité à distance, interface famille-État-nation

\section{Pour citer cet article :}

Mummert, G. (2013). Familles transnationales défiant les États-nation : quatre lieux d'engagement autour des droits des enfants et des familles. Alterstice, 3(1), 17-34. 


\section{Introduction}

Cet article $^{1}$ porte sur le quotidien des familles transnationales, ces groupes de parents qui organisent leurs activités productives et reproductives à travers une ou plusieurs frontières internationales et vivent face à plus d'un Étatnation. Leur vie, au jour le jour, est marquée par l'incertitude, le sacrifice et la fluidité, entre les contraintes dictées par les gouvernements et les opportunités créées par les sujets transnationaux eux-mêmes qui se voient dotés par les États-nation d'un statut ambigu où ils font l'objet de soupçons permanents. Pour arriver à une meilleure compréhension de ce vécu particulier, dans notre analyse des rapports tissés entre les familles transnationales et les États-nation, nous voulons remettre en question le stéréotype d'institutions d'État qui surveillent constamment les activités et les pratiques de leurs citoyens et résidents, ceux-ci se retrouvant comme victimes passives des contrôles de celles-là, et souligner, au contraire, les actions menées par les membres des familles transnationales qui, en naviguant à travers les interstices légaux entre deux gouvernements, vont jusqu’à défier leur autorité.

L'article, divisé en trois parties, commence par un aperçu sur la croissance et l'énorme diversité de familles transnationales autour du monde et sur la complexité de leurs voies de formation et de leurs dynamiques internes. Loin d'être simplement le résultat de décisions individuelles, ce type de familles est issu des tendances globales de mobilité et de restructuration économique et politique, de programmes de recrutement de la main d'œuvre, voire de persécutions et de conflits armés. Autrement dit, comme l'a montré Parreñas (2000), elles constituent les solutions "temporaires » prises face au désespoir ressenti par des millions de mères et de pères autour du globe qui ne peuvent subvenir aux besoins matériels de leur famille qu'en travaillant ailleurs, en se séparant physiquement de leurs enfants. Dans d'autres travaux (Mummert, 2010), nous avons passé en revue quelques expériences de diasporas, généralement des pays du Sud vers les pays du Nord et de l'Est vers l'Ouest afin de soutenir notre position : l'essor de cette forme d'organisation de la vie en famille est dû aux flux de déplacement et est imposé par les mécanismes de recrutement d'un certain type de main-d'œuvre par les entrepreneurs capitalistes, appuyés par leurs gouvernements ${ }^{2}$.

Dans la seconde section, pour illustrer quelques-uns des enjeux que représentent les familles transnationales pour les États-nation, nous analyserons quatre sites d'engagement - s'enchaînant depuis la Guerre froide dans les années 1960 jusqu'au "nouvel » ordre économique et politique mondial du XXI siècle (voir chronologie en annexe A). Nous verrons que ces enjeux concernent, dans un premier plan, les soins quotidiens aux enfants, aux personnes âgées et aux malades, mais aussi la citoyenneté et les identités multiples, voire les engagements sociaux en faveur des droits des hommes, des femmes et des enfants, et surtout des migrants.

Le premier cas, dans l'ordre chronologique, traite du plus grand exode infantile d'Occident au XX ${ }^{\mathrm{e}}$ siècle. Connu comme l'Opération Peter Pan ${ }^{3}$ et mené de 1960 à 1962, ce plan secret, appuyé par la CIA, a réussi à faire sortir de Cuba - au début du régime communiste de Fidel Castro - 14048 mineurs cubains et les faire entrer aux États-Unis avec un visa spécial. Leurs parents ont envoyé ces enfants (garçons et filles) seuls au pays voisin en considérant cette acte comme un "sauvetage " temporaire contre l'endoctrinement soviétique et en pensant qu'ils pourraient les rejoindre sous peu, une fois qu'ils auraient obtenu le permis de sortie de la part des autorités cubaines. Or, quand la frontière est devenue infranchissable lors de la suspension des permis par Castro, ils n'ont pas pu les rejoindre et, en réalité, la majorité des enfants Peter Pan a grandi sans père et mère.

\footnotetext{
${ }^{1}$ L'auteure tient à remercier Catherine Bony et Anna Olivier pour la révision du texte en français et María de la Luz Arceo Flores, Victoria Luna Velarde et María del Carmen Moreno Cárdenas pour la systématisation des données des quatre cas d’études. Elle reconnait aussi la valeur des commentaires critiques des évaluateurs anonymes et de Nadia Guessous.

2 De façon parallèle, Newman (2012) analyse, pour six pays industrialisés, l'essor récent de la forme familiale plurigénérationnelle dite " accordéon » (où les enfants devenus adultes restent ou bien retournent chez leur parents) comme produit des conditions des marchés de travail et des politiques gouvernementales, ce qu'elle appelle dans son titre " les dégâts privés de la compétition globale ».

${ }^{3}$ La presse (suivant peut-être la $\mathrm{CIA}$ ) a baptisé la mission sous ce nom, faisant allusion à une pièce de théâtre de l'Écossais Barrie qui raconte, en 1904, I'histoire d'un petit garçon qui ne voulait pas grandir et pouvait voler. Les Cubains ont traduit Peter par Pedro (Gay, 2000, p. 5-6).
} 
Le deuxième cas se déroule en 1999 et 2000, quand Elián González, enfant cubain de cinq ans, fait naufrage près des côtes de Floride et est trouvé seul, attaché à une bouée flottant sur l'océan. Étant donné que sa mère s'est noyée en essayant d'entrer par barque aux États-Unis, sans papiers, avec d'autres Cubains, la présence d'Elián sur le territoire des États-Unis a déchaîné un délicat cas diplomatique et une dispute légale où se confondent les droits de l'enfant, ceux de son père resté à Cuba et les désirs de sa famille élargie anti-castriste émigrée à Miami.

Le troisième cas est celui d’Elvira Arellano, mère célibataire mexicaine qui a lutté pendant six ans (2002-2007), au nom de son fils citoyen étatsunien, contre un ordre de déportation issu de la Cour des États-Unis. Ayant adhéré au mouvement "Sanctuaire " ${ }^{4}$ des sans-papiers, elle est restée une année entière dans une église de Chicago, posant à plusieurs reprises cette question embarrassante : l’État a-t-il le droit de séparer la famille?

Le dernier cas concerne les orphelins du tremblement de terre qui a eu lieu à Haïti, le 10 janvier 2010, provoquant une mobilisation de l'aide internationale de la part des gouvernements des pays du Premier Monde, des organisations supranationales et des agences non gouvernementales. Les essais d'adoption internationale des enfants haïtiens ont déchaîné une vive querelle, qui a mis en cause la position des parents adoptifs potentiels qui considéraient que cette voie était "la bonne solution " pour les mineurs, surtout dans cette situation extrême de désastre et de chaos régnant dans le pays le plus pauvre de l’Amérique Latine.

Il existe déjà des analyses de chaque cas séparé ainsi que d'autres qui tendent des ponts entre l’Opération Peter Pan et le cas Elián ${ }^{5}$. Pour le premier, les auteurs sont les enfants mêmes qui ont été " sauvés » et qui, devenus la plupart professeurs d'université, ont écrit leurs mémoires. Nous reprendrons ici ces études, mais le regard original qu'offre cet article porte sur la recherche d'un lien entre ces quatre événements historiques en tant que lieux d'engagement où les familles transnationales et plusieurs États-nation lancent des discours, entreprennent des actions et luttent pour que prédomine leur définition de " l'intérêt supérieur de l'enfant ». Évidemment, cet intérêt se mêle, du côté des pères et mères, à beaucoup d'émotion et, du côté des acteurs représentant les gouvernements et associations, à toute une série d'intérêts politiques, économiques et culturels. La notion d'interface sociale proposée par le sociologue anglais Norman Long (1989, p. 2) est très utile pour délier ce mélange curieux d'intérêts : "Un point critique d'intersection ou lien entre différents systèmes sociaux, champs ou niveaux d'ordre social où se trouvent le plus souvent des discontinuités structurelles, basées sur des différences de valeur normative et intérêt social " ${ }^{6}$. II précise : "Le concept suppose quelque type de rencontre en tête-à-tête entre des individus ou des noyaux représentant différents intérêts et soutenus par différentes ressources. " Ainsi, en intégrant les quatre cas dans une analyse comparative d'interface, nous insisterons sur les rencontres entre les membres d'une même famille ainsi qu'entre ceux-ci et les organisations non gouvernementales et les représentants des États-nation.

En conclusion, nous proposerons, à partir de ces quatre cas très complexes et liés entre eux, des pistes pour déchiffrer les interfaces entre familles transnationales et États-nation, en offrant une interprétation des disputes qui surgissent autour des soins aux membres de la collectivité censés être vulnérables. Comme le montre le photojournaliste et activiste en faveur des immigrants David Bacon (2008, chapitre 7), historiquement, l'État-nation a surveillé les activités et les pratiques des personnes résidant sur son territoire. Ce faisant, il classifie les personnes en deux catégories : les humains ayant droit (de citoyenneté, de travail ou de résidence) et les humains sans droits. Cette classification, qui a pour racine les diverses formes d'esclavage, se réalise à partir de critères raciaux. II n'y a qu'un pas à faire pour franchir la ligne entre séjour légal/illégal, entre personne ou travailleur légal, en règle, et personne illégale, sans papiers. Finalement, nous partirons des défis qu'affrontent les familles transnationales ainsi

\footnotetext{
${ }^{4}$ Le Mouvement Sanctuaire est né en mars 1982, à Tucson (Arizona), quand un groupe de protestants a protégé des refugiés politiques centraméricains, en les accueillant dans leur église au défi des lois d'immigration du gouvernement étatsunien. Des personnes catholiques et juives devaient faire écho plus tard de cette manifestation.

${ }^{5}$ Voir pour Peter Pan : Conde (1999), Eire (2003 et 2011), Torres (2003), Triay (1999) et Walsh (1971); pour Elián : Bardach (2002); pour Elvira : le film Elvira (2009, voir note 15); pour les orphelins haïtiens : voir Joyce (2013) et plusieurs sites Internet cités dans les références. Gay (2000) et De la Campa (2000) lient explicitement Peter Pan et Elián.

${ }^{6}$ Toutes les traductions de l'anglais de cet article sont de l'auteur.
} 
que des opportunités qu'elles créent pour recommander des actions ayant trait à l'éducation et à la recherche interculturelles.

\section{L'essor et la diversité des familles transnationales}

Tout d'abord, il nous faut expliciter notre définition de famille transnationale : un groupe de parents qui organisent leurs activités productives et reproductives à travers une ou plusieurs frontières internationales et vivent entre plus d'un État-nation. Souvent stigmatisée pour avoir " abandonné » sa responsabilité de protéger et de faire grandir ses enfants, la famille transnationale offre une solution - ni meilleure, ni pire que d'autres - en réponse aux besoins du groupe domestique. Ce qui distingue ce type de famille, c'est le fait qu'elles traversent des frontières politiques entre pays et situent leurs options de prise de décision sur un damier qui n'est pas limité par une ligne de division (le fait de voir à travers ces frontières). Celles-ci sont sans doute contraintes dans leurs actions par les régulations de deux ou plusieurs États-nation mais, en même temps, elles sont actives dans leur recherche d'opportunités pour contourner la loi, pour jouer la carte de plusieurs identités, voire nationalités. Elles sont classifiées par les agences de l’État, mais résistent aussi à ces étiquettes. Par exemple, dans leur caractérisation des familles transnationales d'aujourd'hui, depuis l'Europe, Bryceson et Vuorela (2002, p. xi) insistent sur «la créativité des gens pour imaginer des voies et manières pour soutenir l'idée d'être famille (familyhood) au milieu de la dispersion des membres autour du monde ».

Quant à leurs divers modes de formation, les familles transnationales sont historiquement le résultat de guerres, déplacements forcés, migrations dites «volontaires " et programmes de recrutement de main-d'œuvre dont les euphémismes de travailleur visitant, guestworker, bracero ou Gastarbeiter sont quelques-uns des plus récents. Considérée comme une solution «temporaire» aux nécessités autant familiales que gouvernementales, la séparation des membres se prolonge souvent pendant des années. Au cœur du phénomène, on trouve un paradoxe vécu comme un dilemme par les parents : loin de fuir leurs responsabilités parentales, ils sentent que la seule manière de faire face aux besoins de leurs enfants est de partir travailler ailleurs, ce qui suppose une séparation physique.

$\mathrm{Si}$, en effet, plusieurs typologies de familles transnationales ont été proposées par des experts, tous en distinguent trois formes ${ }^{7}: 1$ ) paternité (le père émigre); 2 ) maternité (la mère émigre) et 3) parentalité (mouvements de père et mère de façon simultanée ou bien décalée dans le temps). Notre révision du corpus d'études à propos de familles transnationales sur quatre continents (Amérique, Asie, Afrique, Europe) ${ }^{8}$ révèle deux faits indéniables : la croissance de la proportion de ce type de familles autour du monde depuis la fin du XX $X^{\mathrm{e}}$ siècle et l'évaluation culturelle clairement différentielle de la maternité et de la paternité. La première, fondée sur la notion que la femme a été dessinée par la Nature pour devenir mère, est plus stigmatisée que la seconde. Comme l'ont montré de façon convaincante des analystes féministes comme Hrdy (1999), les mères sont jugées et se jugent ellesmêmes en fonction d'un modèle de maternité intensive qui naturalise comme travail féminin les soins aux enfants, aux personnes âgées et aux malades. Au contraire, les pères étant censés manquer d'instinct pour soigner les enfants, ceux qui sont absents sont généralement relevés de leurs responsabilités des soins quotidiens, considérés comme incompatibles avec leur rôle de pourvoyeur. Toutefois, certaines mères transnationales - comme les Mexicaines et Centraméricaines travaillant dans la cité globale de Los Angeles et étudiées par Hondagneu-Sotelo et Avila (1997) - ont brossé de nouvelles manières de comprendre ce que signifie être pourvoyeuse/travailleuse loin de ses enfants.

\footnotetext{
${ }^{7}$ L'une des pionnières des études sur les familles transnationales philippines, Rhacel Salazar Parreñas (2000 et 2005), fait un louable effort pour ôter les stigmates en choisissant des étiquettes qui ne soient pas tendancieuses : Mother away (mère à distance), father away (père à distance), mother and father away (mère et père à distance).

${ }^{8}$ Bien qu'il ne soit pas possible de passer en revue dans l'espace de cet article une littérature si vaste, il faut au moins signaler que la plupart des études traite des diasporas de certains pays asiatiques dont la Chine (Pieke, Nyiri, Thuno et Ceccagno, 2004; Rodriguez Garcia, 2011 et Ye, Murray et Yihuan, 2005), les Philippines (Parrenas, 2000 et 2005) et la Corée, de pays latinoaméricains dont le Mexique (Barndt, 2002; Bustamante et Aleman, 2007; Dreby, 2006 et 2010; Farr, 2006; Hellman, 2008; Hirsch, 2003; Mummert, 2009), le Guatemala (Artico, 2003), le Salvador, le Honduras (Nazario, 2007) et l'Équateur (Pribilsky, 2007), de pays européens dont l'Espagne (Pedone 2003) et certains pays de l'Est et enfin de pays africains dont le Sénégal, le Ghana (Coe, 2008), le Maroc, la Tunisie et l'Algérie.
}

Alterstice - Revue Internationale de la Recherche Interculturelle, vol. 3, $n^{\circ} 1$ 
Les études sur la formation, la morphologie et la dynamiques des familles transnationales sont utiles, même si la nôtre est ciblée davantage sur la réinvention quotidienne d'être famille " en dialogue avec » et située parmi plusieurs États-nation. Celles-ci fournissent en ce sens au moins trois pistes intéressantes pour approfondir les analyses anthropologiques des enjeux que représentent la maternité et la paternité pour les États-nation. D’abord, dans les familles transnationales, les liens de connexion que sentent les membres sont exaltés, invoqués, tendus, prêtés, niés, étirés jusqu'à un point de rupture. Le sujet transnational est toujours soupçonné par exemple d'être plus loyal envers un pays qu'envers un autre et, à la limite, obligé de confesser de quel côté il choisirait de lutter en cas de guerre entre ses deux pays. La suspicion existe également en ce qui concerne les questions du droit d'élire et d'être élu et la citoyenneté, qui comprend un faisceau de droits et d'obligations et plus profondément encore de sentiments d'appartenance. Donc, la famille transnationale constitue un cas extrême très révélateur quant aux normes qui régissent les rapports généalogiques, d’un côté, et les négociations possibles ou interdites avec les institutions d'État, de l'autre.

Deuxièmement, en mobilisant une gamme très étendue de liens, les familles transnationales tissent un réseau très complexe de sujets sociaux concernés par la garde des enfants : géniteurs, aides naturelles, frères et sœurs, autres parents, organisations bénévoles et, bien sûr, agences gouvernementales. À l'heure de décider à qui est le tour d'agir, nous pouvons saisir les discours appuyant la naturalisation de certains membres féminins comme soigneuses idéales ou alors les considérables efforts de la part des uns pour convaincre les autres d'agir pour sauvegarder "l'intérêt suprême de l'enfant ».

Troisièmement - et c'est le plus important pour nos objectifs dans cet article -, les familles transnationales nous conduisent en tant qu'analystes précisément aux lieux d'engagement entre l'État et les personnes auxquelles il donne un statut, qu'il protège et éventuellement exclut. Comme l'a montré Minot (2000, p. 3) dans son exposé sur la discrimination envers les parents lesbiens, gays, bisexuels ou transsexuels, jugés " anormaux » par la majorité des personnes, les enjeux sont majeurs, car la plupart des cultures, de même que les conventions et traités internationaux, accordent un respect particulier au lien parent-enfant. Les États-nations répètent souvent des phrases pieuses à propos du caractère sacrosaint de cette connexion. À travers les rapports parentaux, la vie se renouvelle et (ce qui est plus important aux yeux de beaucoup de gouvernements) des valeurs sociales préférées peuvent être transmises et renforcées. Pourtant, cette centralité donne aux États un investissement particulier dans la supervision et contrôle de la parentalité ${ }^{9}$.

\section{Quatre sites d'engagement - de la guerre froide au nouvel ordre économique et politique mondial du $\mathrm{XXI}{ }^{\mathrm{e}}$ siècle}

Voyons maintenant quatre cas qui illustrent notre réflexion sur la famille transnationale comme lieu d'engagement. Il ne s'agit pas de donner des détails sur chaque cas puisqu'il existe d'autres études - la plupart sous forme de témoignage personnel - ayant suivi avec beaucoup de pertinence ces instances dramatiques de familles transnationales qui ont attiré l'opinion publique, les caméras des médias et les réactions des gens communs dans les réseaux sociaux dont ceux d'Internet. Notre objectif est de recadrer ces drames familiaux dans le contexte de l'interface entre groupes qui débattent finalement le sens de "l'intérêt supérieur de l'enfant " inscrit dans les codes juridiques des États-nation et dans l'article 3 de la Convention internationale des Droits de l'Enfant promue en 1989.

\section{L'Opération Peter Pan, un "sauvetage de l'enfance cubaine »}

Ce plan secret fut mené de 1960 à 1962 par l'Église catholique, appuyée par la CIA. II faut souligner que l’Opération Peter Pan n'a pas été fondée sur l'adoption mais qu'au contraire l'Église catholique de l'Archidiocèse de Miami a organisé la sortie de l'île avec des visas spéciaux (dits waiver ou d'étudiant) auprès du Département d’État pour que les enfants (âgés de 6 à 17 ans) puissent voler vers les États-Unis et soient placés provisoirement chez leur

9 J'adopte l'usage du terme parentalité pour me référer à l'exercice des responsabilités de la part des deux parents, mère et père. 
parentèle ou, à défaut de celle-ci, chez des familles d'accueil. Celles-ci étaient pour la plupart catholiques, mais certaines étaient aussi protestantes ou juives.

L'envoi des enfants était considéré comme une solution temporaire en attendant que leurs parents puissent sortir aussi de Cuba pour les rejoindre. Or la plupart des enfants ont grandi en réalité chez des oncles, cousins ou amis, d'autres avec des personnes complètement inconnues, et une autre partie dans des orphelinats. Suite à la suspension des vols commerciaux après la crise des missiles nucléaires soviétiques pointés sur les États-Unis depuis I'île (octobre 1962), beaucoup de parents des enfants Pedro Pan, appartenant surtout aux classes moyennes et supérieures, de race blanche et opposées au régime de Castro, se sont retrouvés sans visa de sortie, captifs sur l'île. Les mesures prises par Castro, dès 1960 (notamment la fermeture des écoles secondaires suivie de l'ouverture de camps à la campagne pour les adolescents dits pionniers, terme emprunté à la révolution socialiste chinoise, ainsi que l'expulsion de religieux catholiques par le régime athéiste), avaient provoqué - parmi ces classes sociales - des craintes d'endoctrinement marxiste-léniniste. Comme l'a documenté le fils de Cubains émigrés, Triay (1999, p. 9), les sentiments de panique et crainte de perte de la puissance paternelle se sont répandus, particulièrement parmi les opposants du régime qui craignaient que des châtiments soient infligés à leurs enfants. "Fidel voulait être le père de mes enfants ", selon l'un d'entre eux (Triay, 1999, p. 1). Une bonne partie de ces parents a décidé de rester dans l'île pour lutter contre Castro en croyant accélérer de cette façon la réunion familiale à Cuba.

Ces enfants, tombés dans l'oubli pendant des décennies et devenus adultes, sont sortis de l'ombre depuis la fin du $X X X^{\mathrm{e}}$ siècle pour raconter et partager leurs expériences. En 1991, ils ont formé une association sous le nom d'Opération Pedro Pan Group (OPPG) ${ }^{10}$. En hommage aux sacrifices des parents des enfants et afin de remercier les États-Unis de leur accueil, ses membres ont pour mission d'aider les enfants vulnérables quels qu'ils soient et de préserver I'histoire de Pedro Pan en essayant de localiser les enfants protagonistes. La création d'une base de données, en mai 2009, par le journal Miami Herald (d'après un registre fait à la main par George, l'homme qui accueillait les enfants à leur arrivée à l'aéroport de Miami) a servi pour que ces presque 14048 personnes puissent se connaître et entrer en contact. Comme nous le verrons plus loin, dans la présentation du quatrième cas, en 2010, certaines figures publiques parmi les membres de l'OPPG ont appuyé l'idée de revivre l'opération pour sauver les orphelins du tremblement de terre à Haïti.

Se déroulant clairement en pleine Guerre froide, cette confrontation idéologique entre les États-Unis et Cuba s'explique du fait que la petite île, localisée à environ 145 kilomètres de son puissant voisin du nord, faisait partie du bloc communiste avec l'Union des républiques socialistes soviétiques (URSS). Pedro Pan est notre point de départ non seulement historique mais aussi analytique car sa taille et sa complexité nous conduisent directement vers un scénario où se joue l'intérêt suprême de l'enfant. Les acteurs sont multiples et leur enchevêtrement exige de nous un effort pour démêler leurs liens, alliances et affrontements. En plus, c'est le seul cas où la presse n'ait pas été autorisée à en parler. Comme on peut l'apprécier dans le schéma en annexe B, ce sont d'abord plusieurs gouvernements qui participent (Cuba, les États-Unis, puis la Jamaïque, la Grande-Bretagne, la Hollande), des organisations religieuses et celles des exilés cubains, installés surtout à Miami. Grâce à l’alliance entre l'Église catholique et les appareils anticommunistes à l'intérieur du gouvernement étatsunien, il a été possible de contourner les procédures normales d'entrée au pays. Dans une deuxième étape, quand la Guerre froide s'est tendue à la suite de la débâcle de la baie des Cochons (avril 1961) et l'embargo qui a interdit le commerce et les vols aériens entre les États-Unis et Cuba (février 1962), il a été nécessaire de se rapprocher de pays amis comme la Grande Bretagne et son ancienne colonie des Caraïbes, la Jamaïque, afin de faire un détour par Kingston, sur la route des États-Unis. Une fois sur le sol étatsunien, il fallait organiser la logistique des soins des enfants. Pour cela, un effort massif baptisé le "Programme pour les enfants cubains " (Cuban Children's Program, ou CCP) a été mis en place et a duré jusqu'en 1981. Ce geste humanitaire, sans précèdent dans le pays, n'est compréhensible, selon l'étude historique de Triay (1999), que dans le cadre des luttes idéologiques de la Guerre froide car c'est la première fois que l'argent de la Fédération a été canalisé vers des agences privées pour s'occuper des soins aux enfants sans parents ni amis aux États-Unis.

\footnotetext{
${ }^{10}$ Le site Internet de leur organisation charitable est : www.pedropan.org
} 
Évidemment, il reste encore beaucoup de questions sans réponse à propos de Pedro Pan. Par exemple, pourquoi Castro a-t-il permis la sortie d'un peu plus de quatorze mille enfants? Est-ce qu'il le considérait comme un chiffre insignifiant, ne représentant que $0,007 \%$ de la population infantile cubaine ${ }^{11}$ ? Pour quelles raisons politiques a-t-il autorisé la publication justement en 2001 (quand notre deuxième cas du "balserito Elián " avait capté à la télévision l'attention du monde entier) d'un livre sur l'Opération Peter Pan dont le sous-titre était « Un cas de guerre psychologique contre Cuba »? En attendant que les documents secrets de la CIA soient dépouillés ${ }^{12}$, nous nous efforcerons maintenant d'analyser les liens entre le premier et le deuxième cas.

\section{Elián González, pion dans la poursuite de la guerre idéologique entre Cuba et les États-Unis}

Naufragé et entré aux États-Unis en tant que "pied sec " quand un pêcheur l'a trouvé en mer et ramené à terre, Elián a d'abord été hospitalisé et ensuite remis par les autorités étatsuniennes à ses parents les plus proches à Miami, un oncle et une cousine de son père. Immédiatement l'enfant s'est trouvé entraîné dans un tourbillon : une dispute familiale complètement idéologique. La position adoptée par son père biologique resté à Cuba était claire: son fils devait retourner chez lui (c'est à dire dans son pays). Juan Miguel González (divorcé de la mère biologique qui lui a enlevé l'enfant sans son autorisation) et Fidel lui-même demandaient le retour de l'enfant. Or les parents exilés de la famille élargie demeurant depuis longtemps à Miami et anticastristes (personnes qu'Elián ne connaissait guère) considéraient que l'enfant devait rester aux États-Unis où il jouirait d'opportunités et de la liberté dont il était privé à Cuba. Ils ont même ressuscité symboliquement la mère en disant qu'elle était morte en cherchant à offrir cette liberté à son fils. Comme Elián est arrivé comme "pied sec » et non pas comme " pied mouillé ${ }^{13}$, une demande d'asile a été présentée par les membres de la famille élargie. Une garde partagée avec le père a été proposée comme solution pour le «balserito », surnom affectueux qu’Elián a reçu.

Dès le début, la dispute s'est jouée non seulement entre la famille séparée par le détroit de Floride et par ses positions idéologiques mais aussi entre les deux pays : Cuba et les États-Unis. À la limite, elle se jouait entre trois nations, comme le suggère De la Campa (2000, p. 3) en utilisant la métaphore d'une nation cubaine déchirée, scindée en deux : I'île et la communauté de Cubains à l'extérieur. Sans doute les rapports ont-ils été tumultueux, marqués par le rapprochement (les États-Unis ont initialement reconnu Castro quand il a chassé le dictateur Bautista du pays en 1959), puis par la rupture des relations diplomatiques et l'embargo commercial (quand Fidel a nationalisé les intérêts de la compagnie United Fruit, entre autres). Or, dans le cas Elián, le père a insisté sur le fait qu'il fallait laisser de côté Fidel et que c'était lui, Juan Miguel, qui s'opposait à la demande d'asile. Les autorités étatsuniennes ont donc considéré que l'intérêt supérieur d’Elian exigeait la réunification familiale à Cuba, mais les parents de Miami ont refusé de le laisser partir.

Les négociations entre la ministre de Justice elle-même et le grand-oncle ont échoué. L'impasse a trouvé une issue grâce à un acte de violence d'État : dans un raid du département de la Justice des États-Unis, au petit matin du 22 avril 2000, l'enfant a été arraché de force des bras de sa famille élargie résidant à Miami et transféré à la capitale de la nation, Washington. Après plusieurs appels devant la Cour promus par les avocats des parents anticastristes, Elián a finalement été remis à son père pour retourner dans l'île, le 28 juin 2000. Ce cas illustre bien la multiplication du nombre de groupes concernés, leurs alliances et affrontements dans la recherche de ce que les uns et les autres essayent de définir, à savoir le bien-être de l'enfant.

\footnotetext{
${ }^{11}$ La population infantile (5-19 ans) selon le recensement de 1953 s'élevait à 1939 300. II n’y a pas eu de recensement en 1960. http: //www.cicred.org/Eng/Publications/pdf/c-c11.pdf (consulté le 17 février 2012).

${ }^{12}$ Plusieurs des auteurs des études sur Pedro Pan ont entrepris des demandes légales auprès du gouvernement étatsunien pour accéder aux documents secrets, sans succès jusqu'à présent.

${ }^{13}$ Cette politique, issue des accords négociés entre les deux gouvernements en 1994-1995, est sujette à interprétation.
} 


\section{Elvira Arellano, symbole des sans-papiers dans l'après 11-septembre}

En 2002, la Mexicaine Elvira Arellano, mère célibataire d'un petit garçon de 3 ans - lui-même né aux États-Unis et donc citoyen - travaillait sans papiers dans les services de nettoyage de l'aéroport O'Hare de Chicago. Elle a été détenue par les autorités Immigration and Customs Enforcement (ICE) pour avoir utilisé un faux numéro de sécurité sociale. Quoique sa situation d'irrégulière ait été découverte, elle savait qu'elle n'était pas seule. Elle s'est érigée en symbole de tous les sans-papiers, exclus des droits humains élémentaires. Et c'est à ce moment-là qu'a commencé sa lutte de six ans (2002-2007) pour rester au côté de son fils aux États Unis (voir schéma en annexe C).

La position du système juridique était que l'enfant (citoyen ayant droit) pouvait rester sous le gardiennage de l'État mais que la mère (sans droit) devait renoncer à la garde de son fils et partir. Comme c'est le cas de beaucoup de familles transnationales, l'État étatsunien leur avait assigné des statuts migratoires différents. Convaincue de son droit, elle s'est appuyée sur le mouvement Sanctuaire et a créé l'organisation La famille latine unie dans un climat anti-immigrant autant aux États-Unis que dans d'autres pays de réception de migrants qui menaient la Guerre contre le terrorisme. Grâce à l'appui des congressistes locaux à Chicago, sa déportation a été déplacée, mais, en 2006, au lieu de se présenter devant le juge, Elvira a décidé de recourir à l'accueil de l'Église Méthodiste de Chicago où elle a trouvé refuge pendant un an. Ce faisant, elle a été étiquetée comme "fugitive » et " criminelle " par l’État étatsunien. Puis, alors qu'elle sortait de son refuge pour assister à une manifestation en faveur des droits des immigrants à Los Angeles, elle a été arrêtée de nouveau le 19 août 2007 et rapidement déportée à Tijuana, du côté mexicain, la même nuit. Son fils Saul est resté sous la garde temporaire d’Emma Lozano, sa marraine, commère rituelle et compagne de lutte d’Elvira.

De la même façon qu'Elián a été utilisé par les deux bandes en dispute, le petit Saul - qui a la double nationalité et pouvait, lui, traverser librement la frontière que sa mère avait l'interdiction de franchir - voyageait entre le Mexique et les États-Unis pour faire des apparitions publiques en soutenant la cause de sa mère. Soit au côté d’Emma, soit accompagné du pasteur Walter Coleman, "Saulito » apparaissait dans les marches, les conférences de presse et les manifestations, s'adressant aux présidents G. W. Bush puis Obama ainsi qu'au Président Calderón au Mexique et au Congrès mexicain en leur demandant de promouvoir des réformes migratoires qui ne sépareraient plus les familles. Son visage a été projeté dans les médias principalement par les groupes activistes en faveur de ces réformes comme " le visage de la déportation » ${ }^{14}$ tandis que celui d'Elvira l'était comme " le visage des droits des immigrants ». En 2008, l'enfant, alors âgé de 9 ans, pris en charge par sa marraine Emma (femme du pasteur de l'Église où Elvira s'était réfugiée), a déclaré qu'il souhaitait être un enfant " normal » mais qu'il devait lutter pour sa mère. Au bout de quelques mois, la mère activiste Elvira a décidé que Saul devait vivre avec elle au Mexique et les deux sont réunis maintenant au Michoacán.

De façon parallèle au cas d'Elián, les médias sociaux et la presse se sont convertis en des lieux d'engagement, une interface sui generis, car ne relevant pas du tête à tête. Ils ont constitué des outils puissants pour mobiliser l'opinion publique en faveur d'Elvira ou contre elle. D'une part, celle-ci a reçu l'appui d'autres immigrants qui chantaient dans une manifestation "Nous sommes tous Elvira », reconnaissant la typicité de son cas tandis que d'autres se montraient passionnément contre, même de façon irrespectueuse en l'étiquetant comme criminelle, prostituée qui se cache derrière son fils et son Église. Le journal Chicago Tribune a fait une enquête auprès de ses lecteurs cybernétiques en posant quatre questions clef sur ce cas. Comme on peut le constater dans les résultats recueillis dans le tableau 1, le sentiment enregistré au lendemain de sa déportation, en août 2007, est clairement contre Elvira et son mouvement. En général, les participants n'étaient surtout pas d'accord avec la comparaison entre Elvira et Rosa Parks, cette femme noire qui en refusant de s'asseoir à l'arrière du bus à Montgomery (Alabama) a déclenché la paralysie du système de transport public dans cette ville du Sud, marquant un moment définitif pour le mouvement des droits civils des Noirs dans les années 1960.

\footnotetext{
${ }^{14}$ Plusieurs journaux étatsuniens et reportages à la télévision ont utilisé ces étiquettes.
} 
Tableau 1 : Résultats d'une enquête d'opinion sur le cas Arellano (21 août 2007)

\begin{tabular}{|c|c|c|}
\hline Questions clefs & Oui (\%) & Non (\%) \\
\hline $\begin{array}{l}\text { Elvira Arellano devrait-elle avoir été arrêtée pour avoir contourné les lois } \\
\text { migratoires des États-Unis? }\end{array}$ & 91,5 & 8,5 \\
\hline - Un bâtiment religieux doit-il être un sanctuaire de la Loi ? & 17,6 & 82,4 \\
\hline $\begin{array}{l}\text { Le fils (8 ans) d’Elvira est citoyen des États-Unis. Est-ce que ceci doit entraîner une } \\
\text { différence dans la façon de traiter son cas de déportation? }\end{array}$ & 23,6 & 76,4 \\
\hline $\begin{array}{l}\text { En laissant de côté vos sentiments personnels, pensez-vous que le cas Arellano } \\
\text { provoquera tellement de sympathie et de passion parmi ses sympathisants qu'elle } \\
\text { deviendra la Rosa Parks du mouvement en faveur des droits de l'immigrant? }\end{array}$ & 15,9 & 84,1 \\
\hline
\end{tabular}

À la différence des deux cas antérieurs, la protagoniste (Elvira) a été durement critiquée par les groupes antiimmigrants, voire par d'autres immigrants. Ces derniers ont critiqué la mère comme porteuse de symbole pour avoir rendu publique sa situation personnelle (être mère célibataire, condition qu'ils considéraient honteuse), pour s'être cachée derrière son fils (position de la Fédération pour les réformes d'immigration américaine, FAIR) et pour avoir eu un bébé "ancre " comme stratégie de permanence (position de Colorado Alliance for Immigration Reform). Selon certains immigrants, elle aurait dû retourner chez elle au Mexique avec son fils et ne pas faire autant de bruit car c'était négatif pour tous - pour d'autres sans-papiers autant que pour ceux qui avaient leurs papiers en règle. II est certain que devant les autorités étatsuniennes et devant l'opinion publique, Elvira a joué la carte de la maternité mais aussi de l'immigrant auquel on a dénié des droits humains élémentaires.

Il est évident qu'Elvira a fortement attiré l'attention de plusieurs segments de la société étatsunienne et mexicaine. Par exemple, un cinéaste mexicain formé à Chicago en a fait un documentaire intitulé " Elvira ${ }^{15}$, le parti politique mexicain de gauche PRD l'a choisie comme candidate congressiste à la ville frontalière de Tijuana (campagne qui a échoué) et les sœurs catholiques Hermandad Mexicana Transnacional - les mêmes qui l'ont accueillie lors de sa déportation - ont ouvert à Tijuana, en décembre 2007, une auberge pour les femmes déportées avec leurs enfants, baptisée "Casa Refugio Elvira ». Dans ce troisième cas, cette femme, qui ne voulait pas rester dans l'ombre ni se taire, a lancé un nouveau mouvement, Sanctuaire, au nom de toutes les familles de statut mixte comme la sienne. Dans ses rencontres avec l'État-nation étatsunien et ses divers représentants, Elvira a défié les ordres d'un système de justice qu'elle considérait comme injuste, défendu un espace familial et exigé une reconnaissance pour les sanspapiers. En ce sens, Elvira constitue un paradigme d'acteur qui - s'appuyant sur des réseaux transnationaux dans la tradition de l'aide mutuelle - a su utiliser au maximum ses ressources limitées pour amorcer un changement dans les rapports de force. Voilà donc une démonstration de la fécondité de l'approche de l'interface sociale.

\section{L'Opération Pierre Pan de nouveau à Haïti?}

Le 10 janvier 2010, l'île d'Haïti, pays le plus pauvre d'Amérique latine et l'un des plus misérables du monde, a été secouée par un fort tremblement de terre de 7.0 degrés sur l'échelle de Richter. Quoiqu'il soit comparé à Peter Pan, le cas est différent, car si les enfants haïtiens en situation vulnérable étaient aussi orphelins, leur nombre s'élevait peut-être à des dizaines de milliers. Même avant la tragédie naturelle, I'UNFPA (Fond des Nations unies pour la population) estimait qu'il y avait 380000 orphelins à Haïti, dont une partie se trouvait en processus d'adoption internationale. Suivie constamment par des reportages, l'intervention humanitaire des agences internationales a invoqué les droits de l'enfant et surtout leur protection. Les États-Unis ont alors offert le statut de temporary protective status aux orphelins et ont annulé les procès de déportation contre les Haïtiens résidant sans

\footnotetext{
${ }^{15}$ Le film Elvira (2009) a été réalisé par Javier Solórzano Casarin, fils d’un journaliste mexicain qui avait interviewé l’activiste.
} 
papiers sur son territoire. Certains pays où des adoptions étaient en cours ont voulu accélérer les démarches à cause des affreuses conditions et du chaos qui régnait à Haïti.

À peine deux jours après le désastre naturel, l'Archidiocèse de Miami a annoncé son projet de copier l'Opération Peter Pan des années 1960 pour faire entrer, cette fois-ci, des orphelins haïtiens (dont les parents étaient morts dans le tremblement de terre) aux États-Unis avec des visas humanitaires. Le Bureau des services légaux catholiques proposait de mettre en place les mêmes mécanismes qui avaient servi aux enfants cubains, cinq décennies auparavant : négocier des visas spéciaux avec le gouvernement des États-Unis afin de pouvoir accueillir les enfants dans des refuges temporaires en Floride. Le projet a été baptisé « Pierre Pan ».

C'est dans cette situation dramatique dont ont témoigné les journalistes que la Croix Rouge Internationale ainsi que d'autres associations d'aide humanitaire et des groupes concernés ont vite pris position. Par exemple, d'un côté, la présidente du Conseil pour la Liberté de Cuba basée à Miami a appuyé l'initiative de l'Archidiocèse, en louant les résultats de Peter Pan. Les adultes qui avaient été eux-mêmes autrefois des enfants Pedro Pan se sont joints à la cause : protéger (sauver d'une vie de souffrance à Haïti) les enfants sans protection. La réponse cubaine dans les réseaux sociaux ne s'est pas fait attendre : une critique féroce était adressée à Peter Pan et un appel à ne pas répéter l'histoire et à arrêter le projet d'arracher les enfants haïtiens à leur pays.

Évidemment, les conditions politiques mondiales avaient beaucoup changé depuis l'apogée de la Guerre froide. L'intervention de certains États-nation (notamment la Hollande et la France) invoquant la protection des enfants a déclenché de forts débats internationaux autour de l'adoption des enfants à risque. La couverture médiatique, l'existence de la Convention des Droits de l'Enfant (signé en 1989) ainsi que de celle de Droits des migrants et des membres de leurs familles (2003) et la crainte de la traite humaine ont créé un nouveau damier où les grandes puissances internationales ne peuvent pas agir aussi impunément qu'auparavant. En 2010, leurs actions "bénévoles » ont été soupçonnées de cacher d'autres intérêts plus « mondains ". L'UNICEF (Fonds des Nations unies pour l'enfance) a même déconseillé l'adoption d'enfants haïtiens comme une solution d'aide, et d'autres organisations non gouvernementales ont exprimé leur crainte que les enfants, sous couvert de sauvetage, puissent tomber entre les mains de criminels.

Malgré ces voix précautionneuses, plusieurs centaines de mineurs haïtiens ont été reçus aux États-Unis. II est devenu clair, au fil du temps, qu'il y en avait parmi eux certains dont les parents biologiques avaient survécu, créant ainsi des situations légales et diplomatiques très complexes, et qui ne sont toujours pas résolues. Dans ces cas, à qui est-ce le tour de définir " l'intérêt supérieur de l'enfant »?

\section{Conclusion : le déchiffrage de l'interface sociale familles - États-nation}

Notre analyse en chaîne des quatre cas révèle la fécondité méthodologique d'une approche diachronique d'interface sociale pour analyser les lieux d'engagement et de lutte entre les familles transnationales et les Étatsnation. Rappelons que la Déclaration universelle des droits de l'homme établit que la famille est l'unité naturelle et fondamentale de la société, par conséquent elle doit être protégée par la société et par l’État (Hearst, 2004). En lisant horizontalement les cas présentés ici, en les voyant ancrés dans les processus mondiaux (voir l'enchevêtrement dans la chronologie), on perçoit «la nature dynamique et potentiellement conflictuelle de l'interface sociale » soulignée par Long $(1989$, p. 1). Par exemple, dans l'Opération Peter Pan et le cas Elián, nous voyons clairement comment différents groupes anticastristes (y compris le gouvernement étatsunien) ont fait alliance pour promouvoir le sauvetage infantile tandis que les autorités cubaines ont répondu par des mesures limitant les droits de mobilité et d'expression de leurs citoyens.

Nous avons tiré plusieurs leçons de cet exercice. D’abord, dans les rencontres, les idées préconçues à propos de la citoyenneté ainsi que les sentiments d'appartenance mobilisés par les différents membres de la famille sont remis en question. Des sujets qui ne sont même pas des consanguins, ni apparemment directement concernés, peuvent s'engager. Dans le cas d'Elián, par exemple, on a vu la sagesse de l'avertissement de Long (1989, p. 223) de chercher «les interactions, négociations, et luttes sociales et cognitives qui se produisent entre des acteurs sociaux spécifiques. Ceux-ci incluent les personnes présentes dans certaines situations en tête à tête mais aussi celles qui 
sont absentes mais néanmoins pèsent sur ces situations, autant les actions que les dénouements ", à savoir la mère noyée et Fidel Castro. Quant à la citoyenneté obtenue par droit de naissance sur le sol étatsunien, faisant partie de l'amendement 14 de la Constitution des États-Unis depuis 1868 (quand elle a été adoptée pour assurer la nationalité des enfants des esclaves libérés), elle est maintenant remise en question par certains conservateurs du Parti républicain et groupes anti-immigrants qui y voient la possibilité de stratégies pour avoir un " bébé-ancre » 16 . C'est précisément cette idée que l'une des critiques faites à Elvira a soutenu, celle de la mère célibataire qui personnifie des images contrastantes: criminelle, prostituée, martyre, fugitive, symbole des sans-papiers, mère souffrante, mauvaise mère. ${ }^{17}$

Nous avons en deuxième lieu montré les effets des médias sur ces interfaces. En suivant dans la chronologie le développement stratosphérique de la télévision (surtout les reportages)et de l'Internet depuis l'après-guerre - des années 1950 jusqu'au début du XXI ${ }^{e}$ siècle - nous avons perçu à quel point la gamme de sujets sur ces thèmes s'est élargie. Les avancées technologiques ont changé la donne, car maintenant les yeux du monde entier se fixent immédiatement sur les actions des États-nation, certains groupes de citoyens organisés et des membres de familles transnationales. Les normes actuelles des droits de la personne partagées par une vaste majorité de membres des Nations Unies ont servi de contrepoids aux actions spécifiques des gouvernements. Minot (2000, p. 3-4) signale qu' " autant les interventions actives des États comme leurs retombées dangereuses dans l'indifférence peuvent être jugées à la lumière des normes des droits humains ». L'engagement s'est joué autrement dans l'Opération manquée de Pierre Pan et dans l’Opération Pedro Pan, alors qu'il n'existait ni Internet ni le web, et que le contrôle des informations par les services secrets était blindé comme s'il s'agissait d'une affaire de sécurité nationale. Les enjeux changent au fils du temps, et il existe par exemple aujourd'hui des acteurs (dont les enfants sauvés euxmêmes) qui voudraient en savoir davantage rétrospectivement sur l'opération Pedro Pan. Ils exigent même au gouvernement étatsunien actuel un accès aux documents des années 1960. Ces réclamations des personnes concernées et des historiens soulignent la nature toujours ouverte d'un dossier historique, le fait qu'il n'ait pas de clôture.

Troisièmement, les cas analysés montrent que les lieux des luttes idéologiques menées par divers acteurs (y compris les agents des États-nation), adoptant l'approche de plus en plus privilégiée des droits de la personne et plus spécifiquement les droits de la famille unie, ceux des migrants et deux des enfants, débordent des espaces publics et diplomatiques pour envahir l'espace domestique, conçu de façon erronée comme un refuge contre les éléments naturels mais aussi contre tous les dangers extérieurs. L'enfant, placé en porte-à-faux sur la division privé/public, est souvent présenté comme quelqu'un qu'il faut défendre à cause de sa faiblesse et de son bas âge, mais il est en même temps projeté comme un acteur en soi, capable d'écrire son histoire. Coe et ses collaborateurs (Coe, Reynolds, Boehm, Hess et Rae-Espinoza, 2011, p. 8) signalent cette double prémisse contradictoire comme l'un des fondements des organisations internationales pour l'enfance et pensent que les adultes arrivent même à coopter la capacité d'agir des enfants. Dans nos cas à l'étude, l'utilisation des déclarations des deux petits garçons Elián et Saulito - action souvent critiquée par la presse - pour faire avancer la position de l'une et l'autre bande d'adultes en est un exemple très clair. D’ailleurs, le regard de plus en plus interdisciplinaire des spécialistes sur l'enfance a donné à ceux-ci une nouvelle piste dans la représentation de leurs expériences de vie ${ }^{18}$.

Finalement, et nous l'avons montré ailleurs (Mummert, 2010) pour quatre pays - Mexique, Équateur, Philippines, Chine -, la maternité et la paternité transnationales sont valorisées différemment par les familles et par les Étatsnation, selon le contexte politique et les rapports de force internationaux. L'adoption de l'enfant - formalisée légalement ou pas - se présente comme une option à prendre ou à rejeter dans les quatre enjeux (et davantage

16 Voir l'article "Citizenship from birth is challenged on the right", New York Times, 6 août 2010. En ligne à: www.nytimes.com/2010/08/07/us/politics/07fourteenth.html?_r=1\&emc=eta1\&pag (consulté le 7 août 2010)

${ }^{17}$ La professeure et dramaturge Ana Elena Puga a exploré ces images dans une mise en scène autour des perceptions publiques d'Elvira et a inventé l'expression " mélodrame des migrants ". http://www.ihc.ucsb.edu/migrant-rights-migrant-melodrama/ (consulté le 14 février 2012).

${ }^{18}$ Pour un exemple d'essai autre d'écoute et de compréhension des enfants, voir l'anthologie de Pufall et Unsworth (2004). Douglas explore la tournure autobiographique pour illustrer "les façons diverses dans lesquelles les souvenirs de la jeunesse son médiatisés ou moulés par les institutions sociales et pratiques culturelles » (2010, p. 23). 
dans le cas des orphelins d'Haïti) et soulève la question de la garde légitime et du devoir de l'État-nation de protéger l'institution de la famille et de veiller sur le bien-être de ses jeunes citoyens. Nous sommes là devant ce que Woodhouse (2004, p. 233) appelle « la bagarre entre les parents et l'État-nation pour déterminer qui aura le pouvoir d'articuler et défendre l'intérêt suprême de l'enfant ».

Notre analyse nous mène nécessairement à faire des recommandations dans le domaine de l'éducation et de la recherche interculturelles. Tout d'abord, il faut ôter leurs stigmates aux familles transnationales dans l'opinion publique, la presse et devant les États-Nation pour leur donner un statut tout aussi valable que d'autres formes d'organisation de la vie familiale. Ceci est particulièrement important dans le cas des mères transnationales, qui vivent souvent leur départ de façon contradictoire et souffrent de regrets à cause d'une naturalisation du rôle féminin les obligeant à nourrir et à soigner d'autres membres de leur famille, surtout leurs enfants.

Au sein des familles transnationales, nous constatons que les rapports de parenté et les sentiments de connexion repoussent les frontières de nos connaissances des dynamiques domestiques. II nous manque des études comparatives sur les dyades peu connues telles que les grands-parents qui prennent en charge (et quelques fois adoptent) leurs petits-enfants, les demi-frères et sœurs issus de plusieurs unions (souvent vivant dans les différents pays), le beau-père ou la belle-mère et le beau-fils ou la belle-fille (produits de familles recomposées). II serait important d'en savoir davantage, afin de découvrir leurs expériences de vie et leurs besoins spécifiques, car ces personnes sont mises à rude épreuve et vulnérables aux tensions ${ }^{19}$.

Finalement, il est impératif de réfléchir sur les manières dont les progrès de la technologie ont promu la création de nouveaux réseaux transnationaux et de nouvelles formes de communication ayant des effets variés sur les lieux d'engagement entre familles et États-nation. La téléphonie cellulaire ainsi qu'Internet ont donné naissance à des associations qui se positionnent des deux côtés de la question des droits des immigrants - d'un côté, des fédérations regroupant des clubs locaux de migrants qui luttent pour défendre ces droits et, de l'autre, des organisations franchement anti-immigrants qui font pression sur les gouvernements, les citoyens et leurs élus.

\section{Références bibliographiques}

Artico, C. (2003). Latino families broken by immigration. The adolescents' perceptions. New York : LLB Scholarly Publishing.

Bacon, D. (2008). Illegal people. How globalization creates migration and criminalizes immigrants. Boston : Beacon Press.

Bardach, A. (2002). Cuba Confidential. Love and vengeance in Miami and Havana. New York: Random House.

Barndt, D. ( 2002) Tangled Routes. Women, Work and Globalization on the Tomato Trail. Lanham, MD : Rowman and Littlefield.

Bryceson, D. et Vuorela, U. (dir.) (2002). The transnational family: New European frontiers and global networks. Oxford : Berg.

Bustamante, J. et Alemán, J. (2007). Perpetuating Split-household Families. The case of Mexican Sojourners in MidMichigan and their Transnational Fatherhood Practices. Migraciones Internacionales, 4(January-June), 65-86.

Coe, C. (2008). The Structuring of Feeling in Ghanian Transnational Families. City and Society, 20(2), 222-250.

Coe, C., Reynolds R., Boehm D., Hess J. et Rae-Espinoza, H. (2011). Everyday ruptures. Children, youth, and migration in global perspective. Nashville : Vanderbilt University Press.

Conde, Y. (1999). Operation Pedro Pan. The untold exodus of 14,048 Cuban children. Londres : Routledge.

De la Campa, R. (2000). Cuba on my mind. Journey to a severed nation. London : Verso.

\footnotetext{
${ }^{19}$ Mummert (2011) explore ces dyades dans les familles transnationales mexicaines.
} 
Douglas, K. (2010). Contesting childhood. Autobiography, trauma and memory. Piscataway, NJ : Rutgers University Press.

Dreby, J. (2006). Honor and virtue: Mexican parenting in the transnational context. Gender and society, 20(1), 3259.

Dreby, J. (2010). Divided by borders. Mexican migrants and their children. Berkeley : University of California Press.

Eire, C. (2003). Waiting for snow in Havana. New York : The Free Press.

Eire, C. (2010). Learning to die in Miami. Confessions of a refugee boy. New York : The Free Press.

Farr, M. (2006). Rancheros in Chicagoacán. Language and identity in a transnational community. Austin : University of Texas Press.

Gay, K. (2000). Leaving Cuba. From Operation Pedro Pan to Elian. Brookfield, CT : Twenty-First Century Books.

Hearst, A. (2004). Recognizing the roots: children's identity rights. Dans P. Pufall et R. Unsworth (dir.), Rethinking childhood (p. 244-261). Piscataway, NJ : Rutgers University Press.

Hellman, J. (2008) The world of Mexican migrants. The rock and the hard place. New York : The New Press.

Hirsch, J. (2003). A courtship after marriage. Sexuality and love in Mexican transnational families. Berkeley: University of California Press.

Hondagneu-Sotelo, P. et Avila, E. (1997). I'm here but I'm there: the meanings of Latina transnational motherhood. Gender and society, 11(5), 548-571.

Hrdy, S. B. (1999) Mother Nature: a history of mothers, infants and natural selection. New York: Pantheon.

Joyce, K. (2013). The child catchers. Rescue, trafficking, and the new gospel of adoption. New York : Public Affairs.

Long, N. (1989). Encounters at the Interface: A Perspective on Social Discontinuities in Rural Development, Wageningen: Wageningen Agricultural University.

Minot, L. (2000). Conceiving Parenthood. Parenting and the rights of lesbian, gay, bisexual, and transgender people and their children. San Francisco : International Gay and Lesbian Human Rights Commission.

Mummert, G. (2011). Todo queda en familia (transnacional): niños mexicanos a cargo de cuidadores alternativos. Dans F. Lestage et M. E. Olavarría (dir.), Parentescos en un mundo desigual. Adopciones, lazos y abandonos en México y Colombia (p. 103-131). México : Miguel Angel Porrúa, Universidad Autónoma MetropolitanaIxtapalapa.

Mummert, G. (2010). La crianza a distancia: representaciones de la maternidad y paternidad transnacionales en México, China, Filipinas y Ecuador. Dans V. Fons, A. Piella I Vila et M. Valdés Gázquez (dir.), Procreación, crianza y género. Aproximaciones antropológicas a la parentalidad (p. 167-188). Barcelona : Promociones y Publicaciones Universitarias.

Mummert, G. (2009). Siblings by telephone. Experiences of Mexican children in long-distance childrearing arrangements. Journal of the Southwest, 51(4), 515-538.

Nazario, S. (2007). Enrique's journey. The story of a boy's dangerous odyssey to reunite with his mother. New York : Random House.

Newman, K. (2012). The accordion family. Boomerang kids, anxious parents, and the private toll of global competition. Boston : Beacon Press.

Parreñas, R. (2000). Migrant Filipina domestic workers and the international division of reproductive labor. Gender and society, 14(4), 560-581.

Parreñas, R. (2005). Children of global migration. Transnational families and gendered woes. Palo Alto, CA : Stanford University Press. 
Pedone, C. (2003). "Tu siempres jalas a los tuyos". Cadenas y redes migratorias de la familias ecuatorianas hacia Europa. Thèse de doctorat, Université Autonome de Barcelona, Barcelona, Espagne.

Pieke, F., Nyiri, P., Thuno, M. et Ceccagno, A. (2004). Transnational Chinese. Fujianese migrants in Europe. Palo Alto, CA : Stanford University Press.

Pribilsky, J. (2007). La Chulla Vida: Gender, migration, and the family in Andean Ecuador and New York City. Syracuse : Syracuse University Press.

Pufall, P. et Unsworth, R. (2004). Rethinking childhood. Piscataway, NJ : Rutgers University Press.

Rodríguez García, D. (2011). Padres astronautas e hijos paracaídas, configuraciones del parentesco entre la población china de Canadá: ¿un modelo reproductivo transnacional? Dans J. Grau, D. Rodríguez et H. Valenzuela (dir.), Parentescos. Modelos culturales de reproducción (p. 261-286). Barcelona : Promociones y publicaciones universitarias.

Torres, M. (2003). The lost apple. Operation Pedro Pan, Cuban Children in the U.S., and the promise of a better future. Boston : Beacon Press.

Triay, V. (1999). Fleeing Castro. Operation Pedro Pan and the Cuban children's program. Gainesville : University Press of Florida.

Walsh, B. (1971). Cuban Refugee Children. Journal of Interamerican Studies and World Affairs, 13(3/4), 378-415. [Miami : Center for Latin American Studies at the University of Miami]

Woodhouse, B. (2004). Re-visioning Rights for Children. Dans P. Pufall et R. Unsworth (dir.), Rethinking childhood (p. 229-243). Piscataway, NJ : Rutgers University Press.

Ye, J., Murray, J.et Yihuan, W. (2005). Left-behind children in rural China. Impact study of rural labor migration on left-behind children in Mid-West China. Beijing : Social Sciences Academic Press.

\section{Sites web consultés}

www.unicef.org/french/emerg/haiti_52642.html

http://blogs.chicagotribune.com/news_columnists_ezorn/2007/08/vote-four-key-q.html

http://www.cubadebate.cu/especiales/2010/01/15/operacion-pierre-pan-haiti/

http://www.guardian.co.uk/world/2010/jan/17/haitian-orphans-airlifted-miami-resettlement

www.jsonline.com/news/opinion/29425389.html

Alterstice - Revue Internationale de la Recherche Interculturelle, vol. $3, n^{\circ} 1$ 
Annexe A. Chronologie situant les cas d'étude dans les processus et événements mondiaux Temps social

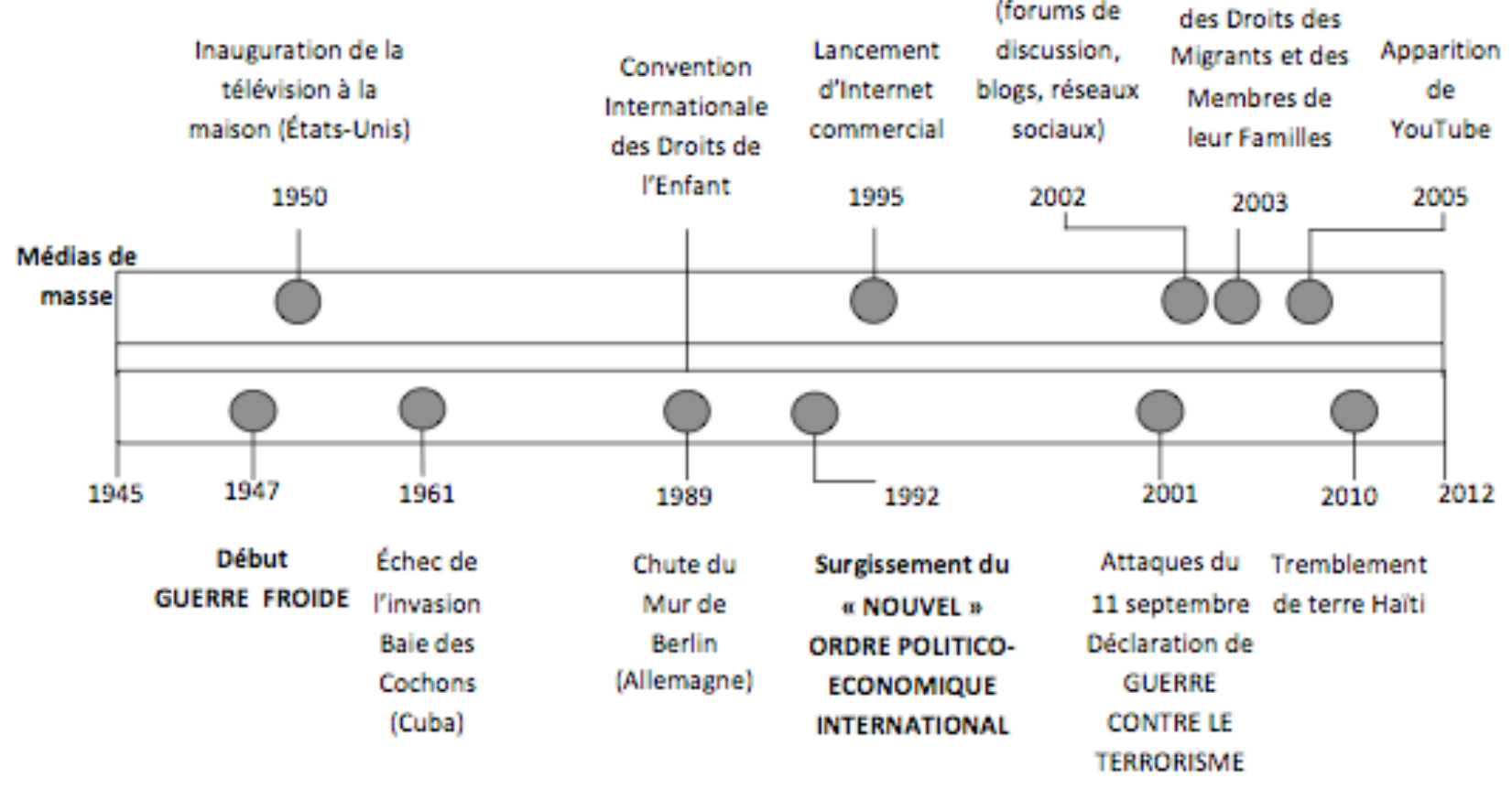

Cas d'étude

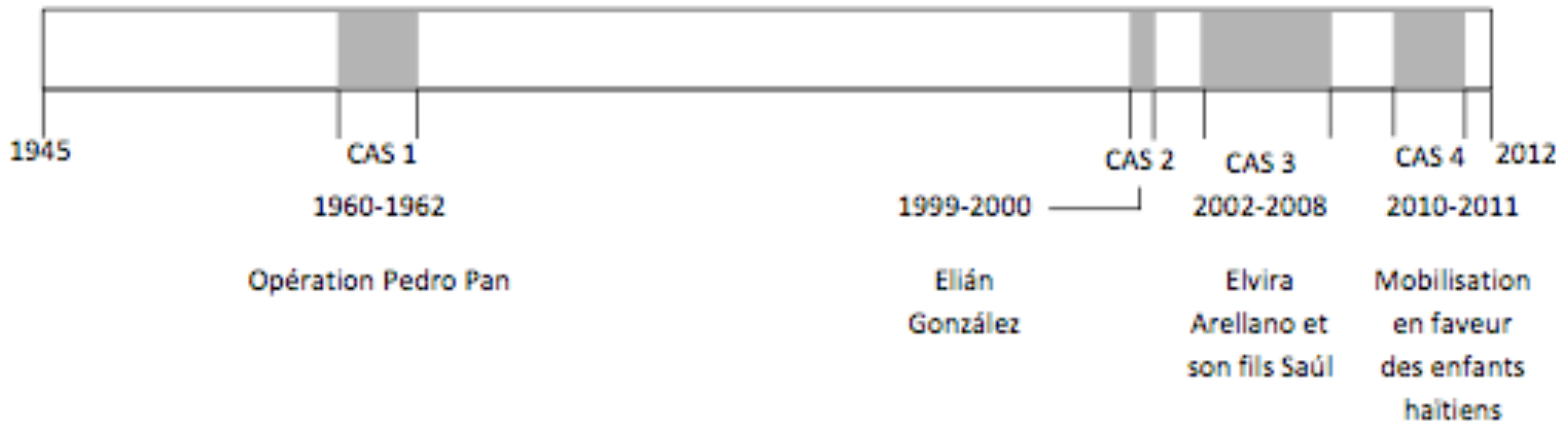


Annexe B. Interfaces sociales pour analyser le cas Pedro Pan

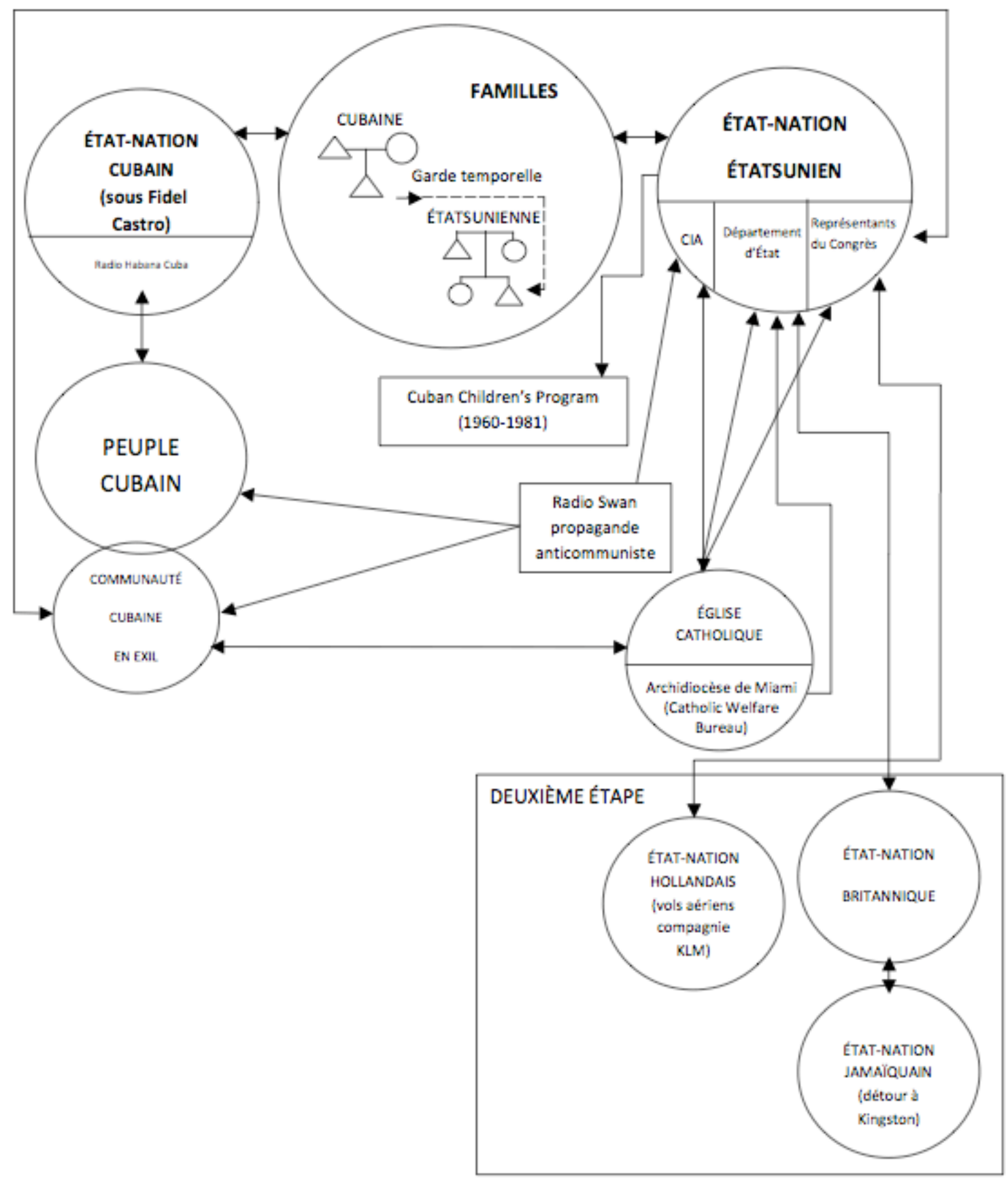


Annexe C. Interfaces sociales pour analyser le cas d'Elvira

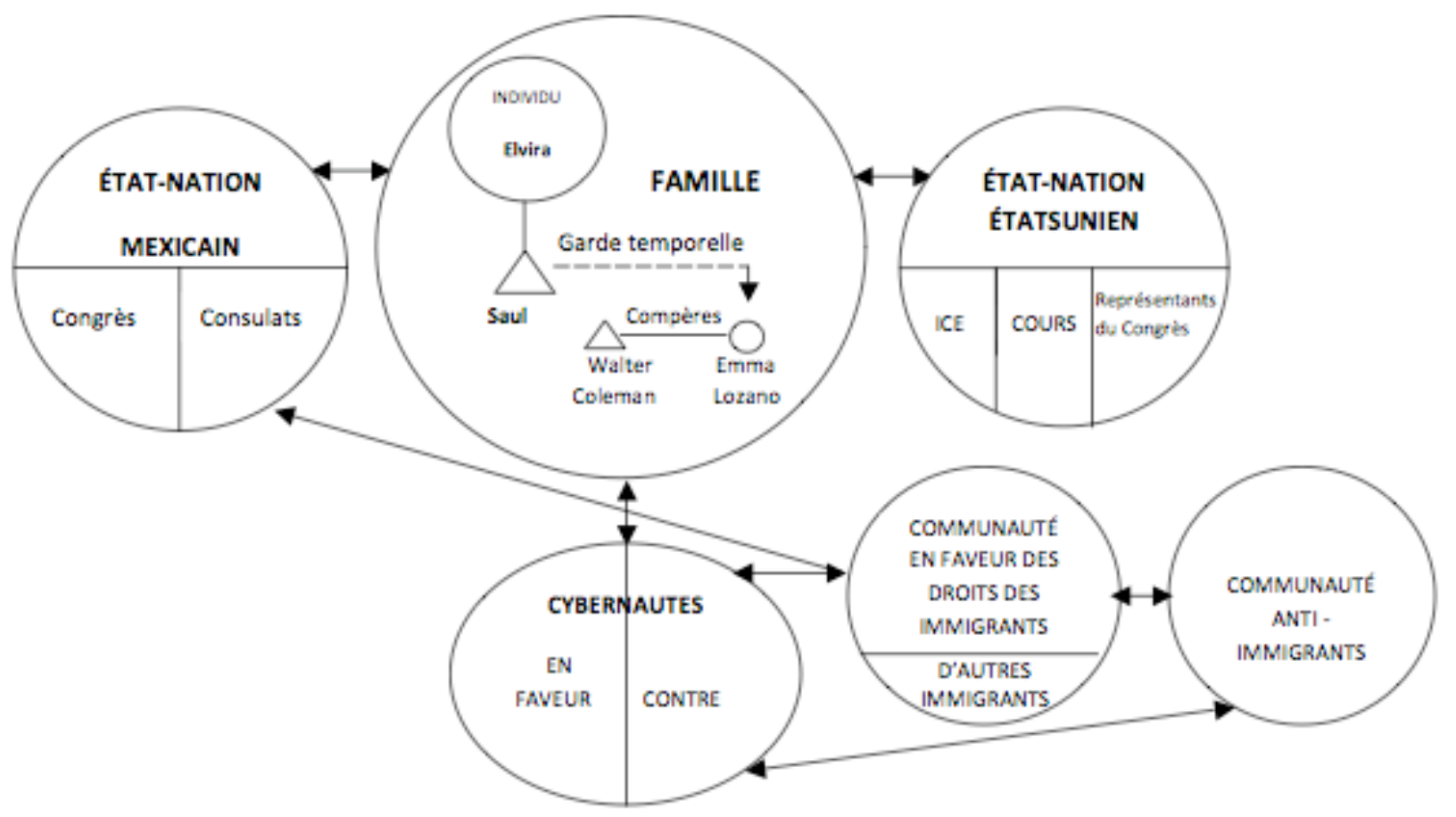

